\title{
Perinatal and Childhood Exposure to Cadmium, Manganese, and Metal Mixtures and Effects on Cognition and Behavior: A Review of Recent Literature
}

\author{
Alison P. Sanders ${ }^{1} \cdot$ Birgit Claus Henn ${ }^{2}$ - Robert O. Wright ${ }^{1,3}$
}

Published online: 5 July 2015

(C) Springer International Publishing AG 2015

\begin{abstract}
Lead $(\mathrm{Pb})$ and mercury $(\mathrm{Hg})$ neurotoxicity is well established. In recent years, a growing body of evidence suggests that environmental exposure to other metals including arsenic (As), cadmium $(\mathrm{Cd})$, and manganese $(\mathrm{Mn})$ and their mixtures also poses public health threats. In this paper, we summarize the recent literature examining the relationship of prenatal and childhood environmental metal exposures with cognitive and behavioral outcomes in children. We conducted a literature search to identify epidemiologic studies that examined the relationship of $\mathrm{Cd}, \mathrm{Mn}$, and metal mixtures with children's neurodevelopmental/cognitive and behavioral outcomes. We restricted the search to peer-reviewed studies published in English between January 2009 and March 2015. We identified a total of 31 articles of which 16,17 , and 16 studies examined the effects of $\mathrm{Cd}, \mathrm{Mn}$, or metal mixtures, respectively. Based on our review, there is suggestive evidence that prenatal/childhood $\mathrm{Cd}$ exposure may be associated with poorer cognition, but additional research is clearly needed. We found little evidence of behavioral effects of early life
\end{abstract}

This article is part of the Topical Collection on Metals and Health

Electronic supplementary material The online version of this article (doi:10.1007/s40572-015-0058-8) contains supplementary material, which is available to authorized users.

Robert O. Wright

robert.wright@mssm.edu

1 Department of Preventive Medicine, Icahn School of Medicine at Mount Sinai, One Gustave L. Levy Place, Box 1057, New York, NY 10029, USA

2 Department of Environmental Health, Boston University School of Public Health, Boston, MA, USA

3 Department of Pediatrics, Icahn School of Medicine at Mount Sinai, New York, NY, USA
Cd exposure, and no studies found a significant relationship with attention deficit hyperactivity disorder. Studies of early life $\mathrm{Mn}$ exposure consistently reported negative impacts on both cognition and behavior. There is also growing evidence that co-exposure to multiple metals can result in increased neurotoxicity compared to single-metal exposure, in particular during early life. Few studies have evaluated behavioral effects related to metal co-exposure.

Keywords Cadmium $\cdot$ Manganese $\cdot$ Metal mixtures · Cognition $\cdot$ Behavior $\cdot$ Childhood

$\begin{array}{ll}\text { Abbreviations } \\ \text { ADHD } & \text { Attention deficit hyperactivity disorder } \\ \text { BSID } & \text { Bayley Scales of Infant Development } \\ \text { CBCL } & \text { Child behavior checklist } \\ \text { CBCL- } & \text { Child behavior checklist - Teachers Report } \\ \text { TRF } & \text { Form } \\ \text { CDIIT } & \text { Comprehensive Developmental Inventory for } \\ & \text { Infants and Toddlers } \\ \text { CVLT } & \text { California Verbal Learning Test } \\ \text { FSIQ } & \text { Full-scale IQ } \\ \text { IQ } & \text { Intelligence quotient } \\ \text { ID } & \text { Intellectual disability } \\ \text { LD } & \text { Learning disability } \\ \text { MSCA } & \text { McCarthy Scales of Children's Abilities } \\ \text { MDI } & \text { Mental development index } \\ \text { NBNA } & \text { Neonatal Behavioral Neurological Assessments } \\ \text { NHANES } & \text { National Health and Nutrition Examination } \\ & \text { Survey } \\ \text { PDI } & \text { Psychomotor development index } \\ \text { PIQ } & \text { Performance IQ } \\ \text { RCPM } & \text { Raven's Colored Progressive Matrices } \\ \text { SDQ } & \text { Strengths and Difficulties Questionnaire }\end{array}$




\section{VIQ Verbal IQ \\ WISC Wechsler Intelligence Scale for Children \\ WPPSI-R Wechsler Preschool and Primary Scale of Intelligence Revised Edition}

\section{Introduction}

Children's exposure to toxic metals such as lead (Pb), arsenic $(\mathrm{As})$, mercury $(\mathrm{Hg})$, cadmium $(\mathrm{Cd})$, and manganese $(\mathrm{Mn})$ is nearly ubiquitous $[1,2]$. These prevalent exposures are a critical public health concern because even relatively low levels of metals, such as $\mathrm{Pb}$, can disrupt normal development of the central nervous system, especially during fetal life and early childhood [3-6]. Environmental contributions to adverse neurodevelopmental and behavioral outcomes in children are a societal burden associated with substantial economic losses [7-9]. For example, the neurotoxic effects of childhood $\mathrm{Pb}$ exposure on decreased intelligence quotient (IQ) are estimated to cost up to $\$ 61$ billion per year in economic losses in the USA [8]. While the neurodevelopmental effects of $\mathrm{Pb}$ and $\mathrm{Hg}$ exposure are well established, and increasing evidence is available for As [10, 11], other metals such as $\mathrm{Cd}$ and $\mathrm{Mn}$ are less well studied, and the economic burden associated with their health effects has not been estimated.

Importantly, metal exposure rarely occurs in isolation and co-exposure is likely the norm $[12,13]$. Metal co-exposure also poses a critical threat to pediatric health [14]. The central nervous system is a common target organ for many environmental metals, leading to the natural question: What happens under joint exposure? Metals may interact to cause synergistic or antagonistic effects on neurodevelopment that are different from the main effects of exposure to each metal alone. Because the number of possible chemical combinations is infinite, researchers must make choices when addressing the role of mixed exposure and health. The epidemiologic literature is now beginning to address lower order interactions between metals, and new methodologies are being developed to address higher order mixtures $[15,16 \bullet]$. Understanding the health effects of combinations of metals, as well as metal interactions with other chemical and non-chemical exposures, is critical for progressing the field of environmental health and protecting children's health [17].

To summarize the state of the literature on the role of $\mathrm{Cd}$, $\mathrm{Mn}$, and metal mixtures as predictors of neurodevelopmental and behavioral outcomes in children, we conducted a review of epidemiologic studies examining early life exposure. We focus on $\mathrm{Cd}$ and $\mathrm{Mn}$ because they are pervasive metals with biological properties that could produce neurotoxic effects and they have not been reviewed recently. We also provide an updated summary of the rapidly growing mixture literature focused on neurodevelopmental outcomes and include a wider range of publication dates than previously reported [14]. We focus on early life, which we differentiate in this paper into two distinct windows - the prenatal period and childhood through adolescence. These life stages appear to be susceptibility windows for environmental metal exposures that place the child at increased risk for subsequent adverse health outcomes, due in part to life-stage-specific developmental processes. We present conclusions based on this body of evidence and provide recommendations for future studies.

\section{Methods}

We conducted a literature search to identify epidemiologic studies that examined the effects of $\mathrm{Cd}$ or $\mathrm{Mn}$ with respect to children's neurodevelopmental/cognitive and behavioral outcomes using the following search terms: (child* OR infan* OR school OR postnatal OR post-natal OR prenatal OR prenatal OR fetal OR pregnan* OR in utero) AND (neurodevelopment* OR cognit* OR mental OR intelligence OR behavior* OR behaviour* OR hyperactivity OR ADHD) AND (metal OR "manganese" [MeSH Terms] OR "cadmium" [MeSH Terms]). To identify studies on metal mixtures, we additionally used the following search terms: (interact* OR effect modif* OR mixture OR joint effect* OR additive effect*). We used PubMed and Web of Science search engines and restricted the search to peer-reviewed human studies published in English between January 2009 and March 2015. We further excluded studies that did not report a cognitive or behavioral outcome (for example, only reported motor effects) or were ecological or semi-ecological in design. Among the mixture literature, we excluded studies that did not report joint or interaction effects. We identified and summarized a total of 16,17 , and 16 studies on $\mathrm{Cd}, \mathrm{Mn}$, or metal mixtures, respectively. For the $\mathrm{Cd}$ - and $\mathrm{Mn}$-specific literature, all reported biomarker concentrations were converted to microgram per liter or microgram per gram to allow comparisons across studies. It should be noted that some studies measured other metals such as $\mathrm{Pb}, \mathrm{Hg}$, and $\mathrm{As}$, but the findings with cognitive or behavioral outcomes are not reported herein unless an interaction effect was presented. Biomarker concentrations were omitted from the mixture literature table for brevity; we direct the reader to the original article for these details.

\section{Sources of Exposure}

In order to fully understand the public health impact of toxic metals, an understanding of their exposure sources is needed. Tobacco is the dominant source of $\mathrm{Cd}$ exposure among smokers; however, the primary source of exposure in the general non-smoking population is diet (seafood, organ meats, grains, leafy vegetables, and root crops) [18, 19]. Anthropogenic sources of $\mathrm{Cd}$ also include industrial smelters, 
phosphate fertilizers, and nickel-cadmium batteries [19]. Although the placenta protects the fetus by restricting $\mathrm{Cd}$, $\mathrm{Cd}$ levels can be detectable in newborn cord blood despite the limited transfer at the maternal-fetal interface [20-22]. Postnatal $\mathrm{Cd}$ exposure can also occur through secondhand tobacco smoke, infant formula [23, 24], or breast milk [25], and the primary source of $\mathrm{Cd}$ in childhood is dietary.

Diet is the primary source of $\mathrm{Mn}$ exposure in the general population (grains, nuts, tea, etc.) [26-28], although only 3$5 \%$ of ingested $\mathrm{Mn}$ is typically absorbed and elimination is tightly regulated through homeostatic mechanisms [29, 30]. Excess exposure most commonly occurs from elevated Mn levels in drinking water or in air sometimes as the result of naturally occurring processes but more commonly due to industrial activities such as welding, mining, steel manufacturing, and combustion of Mn-containing gasoline additives [28]. Mn, like a host of metals including $\mathrm{Cd}$, can be bound to particulate matter, such as particulate matter less than $2.5 \mu \mathrm{m}$ in diameter $\left(\mathrm{PM}_{2.5}\right)$ found in ambient pollution. $\mathrm{Mn}$ is also a component of two extensively applied agricultural fungicides - maneb and mancozeb [31, 32]. Because $\mathrm{Mn}$ is an essential element, it is actively transported across the placenta from mother to fetus [33], which results in higher levels in cord blood than in maternal blood [34, 35]. Postnatal Mn exposure can occur through breast milk [36] and infant formula [37]. Because $\mathrm{Mn}$ is processed and excreted in the liver, oral exposures are per dose less toxic than ambient air exposure. Inhaled Mn aerosols from showering may contribute to exposure [38,39], though primary sources of exposure during childhood and adolescence are diet and drinking water.

\section{Epidemiologic Evidence for Neurotoxic Effects of Cadmium}

\section{Cognitive Effects}

We identified ten studies that examined early life Cd exposure and neurodevelopmental or cognitive outcomes (Supplement 1) [40-49]. Of these studies, five were prospective, four were cross sectional, and one was a secondary analysis of a randomized clinical trial design. Exposure to $\mathrm{Cd}$ was measured at multiple life stages: Prenatal exposure was examined in seven studies [40, 43-47, 49], early childhood exposure (1 to 3 years of age) was measured in one study [41], and two studies evaluated exposure in school-age children (6 to 15 year olds) [ 42 , 48]. A single longitudinal study measured exposure prenatally and at 5 years [47]. Cd levels were measured in blood $(n=6)$, urine $(n=4)$, hair $(n=1)$, and placenta $(n=1)$. Among these, prenatal $\mathrm{Cd}$ exposure was estimated with cord blood $(n=3)$, maternal blood during pregnancy $(n=2)$, maternal urine $(n=$ $2)$, and placenta $(n=1)$. A single study measured both urine and hair and reported significant associations with urine, but not hair Cd levels [48]. Another study measured prenatal $\mathrm{Cd}$ in three matrices - maternal blood at delivery, placenta, and cord blood - and reported significant associations only with cord blood levels [40]. Five studies examined the relationship between Cd biomarkers and IQ [41, 45, 47-49], and five studies examined other neurodevelopmental outcome measures.

Four of five studies identified a significant inverse relationship between prenatal/childhood Cd and IQ [45, 47-49]. A prospective study of 1305 mother-child pairs identified significant inverse associations between maternal first trimester urine $\mathrm{Cd}$ and child full scale, verbal, and performance IQ at 5 years; associations with concurrent child urine $\mathrm{Cd}$ were weaker but still significant [47]. In a prospective cohort of 106 children, a significant inverse association with performance and full-scale IQ at 4.5 years was observed among children with high (>median) cord blood Cd [49]. A prospective cohort of 119 mother-child pairs found a significant inverse association between maternal blood $\mathrm{Cd}$ measured in early pregnancy and performance IQ at 5 years [45]. In a cross-sectional study of 2616 to 9 year olds, urine $\mathrm{Cd}$ but not hair Cd was inversely associated with verbal comprehension on the Wechsler Intelligence Scale for Children (WISC)IV (boys and girls) and with full-scale IQ in boys only [48]. Lastly, a longitudinal study of 441 children identified suggestive trends of inverse relationships between 2-year blood $\mathrm{Cd}$ levels and IQ at 5 and 7 years, although these associations were not statistically significant [41].

Five studies examined associations between $\mathrm{Cd}$ exposure and other neurodevelopmental outcome measures. Prenatal [46] and early life [41] Cd exposure was examined in relation to scores on the Mental Development Index (MDI) of the Bayley Scales of Infant Development (BSID), but no significant relationship was found. A prospective cohort study of 4 year olds in Spain found a suggestive but non-significant association between prenatal $\mathrm{Cd}$ and the McCarthy Scales of Children's Abilities (MSCA) measure of cognition [43]. Two studies identified a significant inverse relationship between cord blood Cd and Apgar score at 1 and/or 5 min [40, 44]. Lastly, a large cross-sectional study in the USA noted significant associations between urinary $\mathrm{Cd}$ levels and learning disorders/disability and special education among 6 to 15 year olds [42]. Taken together, these recent studies on IQ and other neurodevelopmental outcomes are suggestive of a potential detrimental effect of early life $\mathrm{Cd}$ on cognitive ability that may also manifest as impaired learning though the epidemiologic evidence is limited.

\section{Behavioral Effects}

We identified nine studies that assessed early life Cd exposure and behavioral outcomes [41-43, 48, 50-54]: Four were cross sectional, two were prospective, two were case control, and one was a randomized clinical trial design. $\mathrm{Cd}$ was measured 
in blood $(n=5)$, urine $(n=3)$, or hair $(n=2)$, predominantly during early to late childhood (5 to 18 year olds). Two studies assessed prenatal $\mathrm{Cd}$ levels in maternal urine or cord blood $[43,52]$, and one measured early childhood exposure ( 2 years of age) [41]. Five studies examined associations between $\mathrm{Cd}$ and attention deficit hyperactivity disorder (ADHD) or ADHD-like behavior [42, 43, 51, 53, 54], and four assessed other behavioral scales $[41,48,50,52]$.

It is notable that none of the five studies that examined the association between early life $\mathrm{Cd}$ and ADHD reported significant associations [42, 43, 51, 53, 54]. Two of the studies were cross sectional, two used a case-control design, and one was a prospective cohort. ADHD was assessed using multiple formats, either by parental report or by administering established ADHD rating scale tests. While three studies had limited sample sizes with less than 100 cases, two were large populationbased studies with relatively large sample sizes $(>300)$ that measured prenatal or concurrent urinary levels of $\mathrm{Cd}[42$, 43]. In the absence of a meta-analysis, the consistent lack of association in the current literature between early life $\mathrm{Cd}$ and childhood ADHD is suggestive, although not conclusive, evidence of a null association.

Two of four studies reported significant findings between prenatal or concurrent $\mathrm{Cd}$ exposure and other scales of child behavior [50, 52]. A prospective study of Belgian children noted a significant increased risk of emotional problems in 7- to 8-year-old boys following prenatal exposure [52]. In a large cross-sectional study in China, hair Cd levels were associated with withdrawn, social, and attention problems among 7 to 16 year olds [50]. However, in a secondary analysis of a randomized clinical trial in the USA, no significant associations were observed between blood Cd levels at 2 years and a battery of behavior and attention tests conducted subsequently at 5 and 7 years [41]. In another cross-sectional study, no significant associations were found between urine or hair $\mathrm{Cd}$ and a battery of behavior and attention tests at 6 to 9 years of age [48]. Overall, while prenatal or school-age $\mathrm{Cd}$ exposure may impact childhood behavior, data are extremely limited and further research is needed.

\section{Epidemiologic Evidence for Neurotoxic Effects of Manganese}

\section{Cognitive Effects}

We identified 14 studies that examined the association between $\mathrm{Mn}$ and neurodevelopmental or cognitive outcomes in neonates or children (Supplement 2) [55-67, 68・•]. Most studies were cross sectional $(n=10)$, and four were prospective cohorts. Mn was measured in blood $(n=10)$, hair $(n=7)$, or drinking water $(n=1)$. Both hair and blood biomarkers were used in three studies, and all of these reported significant associations with neurodevelopmental outcomes for hair but not blood Mn [63, 65, 66].

All four of the prospective studies reviewed support the notion that the prenatal and early postnatal period may be a sensitive developmental window for Mn exposure with regard to neurodevelopment $[58,61,67,68 \bullet \cdot]$. In a large prospective cohort study of neonates from China, a cord serum Mn threshold of $5 \mu \mathrm{g} / \mathrm{L}$ was identified, above which 3 day olds showed cognitive deficits as measured by the Neonatal Behavioral Neurological Assessment (NBNA) [67]. However, there are well-known limitations to assessing neonates, given the limited neurologic repertoire inherent in newborns. Two studies reported an inverted Ushaped association between blood Mn levels measured at delivery or at 12 months of age and mental development scores on the BSID at ages 6 to 12 months $[58,68 \cdot \bullet]$, indicating the potential for $\mathrm{Mn}$ to act as both an essential element and a toxic metal. Chung et al. reported a transition from beneficial to adverse effects on 6-month mental development scores when maternal blood Mn levels were $24-28 \mu \mathrm{g} / \mathrm{L}[68 \bullet \bullet]$. Finally, a prospective cohort study of 2-year-old children in Taiwan reported that cord blood Mn was associated with decrements in cognitive and language subscales of the Comprehensive Developmental Inventory for Infants and Toddlers (CDIIT) [61]. Although additional study is needed, these findings collectively suggest that prenatal and early postnatal Mn exposure might be a particular concern for early childhood development. If a toxic threshold during pregnancy is confirmed, guidance levels of environmental or dietary Mn exposure during pregnancy should be studied.

The majority (12 of 14) of studies examining childhood $\mathrm{Mn}$ exposure observed significant inverse associations with IQ or other mental development indices (BSID, CDIIT, etc.) [56-61, 63-67, 68••]. These studies were conducted in various geographic locations and populations, had moderate sample sizes (many with $n>200$ ), and adjusted for important covariates (e.g., maternal education, child age, child gender, and nutritional status). Two cross-sectional studies in adolescents that measured blood Mn reported no significant association with IQ [55, 62], although one of these reported significant associations with deficits on the Learning Disability Evaluation scale (LDES) [55]. Another cross-sectional study found that hair Mn, but not blood Mn, was associated with long-term memory and learning on the Children's Auditory Verbal Learning Test (CAVLT) among 7 to 11 year olds [66]. Despite a limited sample size $(n=60)$, a cross-sectional study of 1- to 4-year-old Uruguayan children reported significant inverse associations of hair $\mathrm{Mn}$ with cognition and language, especially among girls, but only in unadjusted models [64]. Taken together, these studies support the conclusion that elevated early life $\mathrm{Mn}$ exposure adversely impacts childhood cognition with particularly consistent associations with IQ. 


\section{Behavioral Effects}

We identified seven studies that examined the association between early life $\mathrm{Mn}$ and child/adolescent behavior [54, 55, 57, 62, 69-71]. The studies used cross-sectional $(n=4)$, casecontrol $(n=2)$, and case-cohort $(n=1)$ designs. One study examined prenatal exposure and six studies assessed exposure in early to late childhood. Five studies assessed Mn exposure using blood, one used hair [57], one measured drinking water Mn levels [71], and one measured Mn in teeth [69]. Three of the studies examined the relationship with ADHD, one examined associations with ASD, and three assessed other behavior or attention scales.

Among the three studies of ADHD, a case-control study in the United Arab Emirates reported increased odds of ADHD with increased blood Mn levels [54]. However, this study had a small sample size ( $n=92$, with only 18 cases of ADHD) and did not adjust for any confounding variables. In a large crosssectional study of South Korean children, blood Mn levels were associated with poorer scores of commission on one of three ADHD tests, but there was no association with doctordiagnosed ADHD [55]. A case-cohort study in Brazil found that the treatment of adolescent ADHD with the common medication methylphenidate (Ritalin ${ }^{\circledR}$ ) significantly reduced blood Mn levels [70]. While it remains unclear whether ADHD medication use potentially affects the concomitant levels of other toxic metals, this is an intriguing finding. A recent study found that methylphenidate administered following chronic postnatal $\mathrm{Mn}$ exposure resulted in improved motor function in rats; however, there was no effect on Mn blood levels [72]. If $\mathrm{Mn}$ metabolism is part of the underlying biologic pathway for ADHD, this finding may be evidence for a biological role of Mn in ADHD causation. Conversely, if methylphenidate alters Mn metabolism independent of its effect on ADHD, this may represent a source of bias. Further research is needed to replicate and understand this relationship.

A case-control study of children in the USA with ASD found that cases had marginally significantly lower levels of enamel $\mathrm{Mn}$, representing postnatal exposure, compared to controls [69]. These findings should be interpreted cautiously, however, because tooth enamel does not track as well as tooth dentine with early life timing of exposure due to its longer maturation process [73]. Nonetheless, the role of teeth as biomarkers for metal exposure deserves further study. Three studies examining scales of adolescent behavior other than ASD or ADHD report conflicting results [57, 62, 71]. A crosssectional study of 11- to 14-year-old Italian adolescents found that blood Mn was not associated with any of the child behavior checklist (CBCL) subscales [62], whereas a cross-sectional study of 8 to 11 year olds in Bangladesh reported a significant association between drinking water $\mathrm{Mn}$, but not blood $\mathrm{Mn}$, with internalizing and externalizing behaviors [71]. A cross- sectional study of 7 to 12 year olds in Brazil found significantly impaired performance on attention when comparing higher hair $\mathrm{Mn}$ tertiles to lower $\mathrm{Mn}$ but did not report a significant linear relationship [57].

Taken together, these studies provide some evidence of a link between early life Mn and ADHD, ASD, or other behavioral outcomes in children but are not conclusive. Future studies should explore the strengths and limitations of various exposure biomarkers, including the use of teeth as a biomarker to retrospectively address exposure timing and more clearly distinguish prenatal versus postnatal exposures. Another knowledge gap is whether medication use alters Mn blood levels directly or as a consequence of therapeutic effects.

\section{Epidemiologic Evidence for Neurotoxic Effects of Metal Mixtures}

\section{Cognitive Effects}

We identified 13 articles that examined metal mixture effects on cognition and/or neurodevelopment (Supplement 3) [16•, 42, 46, 60-62, 74, 75, 76•, 77-80]. Among these studies, six were cross sectional, six were prospective, and one was retrospective. Exposure to metal mixtures was measured at various life stages: Prenatal exposure was examined in six studies $[16 \bullet, 46,61,77,79,80]$, early childhood exposure ( 1 to 3 years of age) was measured in two studies [74, 76•], and the remaining five studies evaluated exposure in school-age children (6 to 15 year olds) [42, 60, 62, 75, 78]. Metal exposure was estimated primarily with biomarkers (blood, hair, and urine), though three studies measured metals in environmental samples (drinking water, air, and soil).

Most studies $(n=11)$ estimated effects of binary combinations of metals on neurodevelopment, even when exposure to more than two metals was measured (e.g., [61, 77]). Most likely, this limitation is a function of sample size and the limited statistical approaches available to examine higher order interactions. $\mathrm{Pb}$ is the most commonly examined metal when assessing interactions with other metals as a mixture: Eight studies considered joint effects of $\mathrm{Pb}$ with $\mathrm{As}, \mathrm{Cd}, \mathrm{Mn}$, or $\mathrm{Hg}[42,46,60-62,74,77,80]$. Lead-Mn interactions were estimated in four studies, three of which reported significant adverse interactive effects. A prospective study of 230 Taiwanese children reported an interaction between prenatal $\mathrm{Pb}$ and $\mathrm{Mn}$ exposure measured in cord blood on scores of the CDIIT at 2 years of age [61]. In this study, in utero exposure to high $\mathrm{Pb}$ and high $\mathrm{Mn}$ was associated with larger deficits in cognition and language development compared to low exposure to both metals or to exposure to high levels of only one metal at a time. In the early postnatal period, a significant $\mathrm{Pb}$ Mn interaction was similarly observed in a cohort of Mexican children [74], where slopes for the effect of 1-year blood $\mathrm{Pb}$ on 
both mental and psychomotor development scores of the BSID-II were steeper among children with high blood Mn levels (highest quintile Mn vs. middle levels). Among 8- to 11-year old Korean children, $\mathrm{Pb}$ exposure was associated with lower concurrently measured IQ scores but only among children with high blood Mn levels [60]. In contrast to the aforementioned three studies, no statistical interaction was found between $\mathrm{Pb}$ and $\mathrm{Mn}$ on IQ in 11- to 14-year-old Italian children, despite considering multiple measures of Mn exposure (blood, hair, air, and soil) [62]. We should note that because this study was cross sectional, it did not have information on early childhood $\mathrm{Pb}$ exposure (average blood $\mathrm{Pb}$ was $1.7 \mu \mathrm{g} / \mathrm{dL}$ at 11 to 14 years of age). Taken together, the evidence to date suggests that Mn may exacerbate the well-documented neurotoxic effects of $\mathrm{Pb}$ among children, particularly at younger ages. Possible mechanisms for this interaction include increased binding affinity for $\mathrm{Pb}$ in the brain in the presence of excess Mn levels [81] or joint interference with the release of dopamine or other neurotransmitters [82, 83].

Interactions between $\mathrm{Pb}$ and $\mathrm{Cd}$ have been examined in two studies $[42,46]$. In a large cohort of 884 mother-infant pairs in Korea, maternal blood $\mathrm{Pb}$ and blood Cd levels measured during pregnancy were related to scores on the BSID-II among 6-month-old infants [46]. Increasing $\mathrm{Pb}$ exposure was associated with lower mental and psychomotor development scores among infants with high (>median) prenatal $\mathrm{Cd}$ exposure, in particular for exposures during late pregnancy. In contrast, no interaction between blood $\mathrm{Pb}$ and urinary $\mathrm{Cd}$ levels was observed in relation to learning disability or special education utilization among 6- to 15-year-old participants of the National Health and Nutrition Examination Survey (NHANES) [42].

Additional evidence that $\mathrm{Pb}$ neurotoxicity may be modified in the presence of other metals comes from a prospective study of $\mathrm{Pb}$ and methylmercury [80] and a retrospective study of $\mathrm{Pb}$ and $\mathrm{As}$ [77]. An antagonistic interaction between prenatal $\mathrm{Pb}$ and $\mathrm{Hg}$ exposure, measured in cord blood, was reported on verbal learning, memory, and attention evaluated in children from the Faroe Islands at 7 and/or 14 years of age, suggesting that high methylmercury exposure may mask adverse $\mathrm{Pb}$ effects. An explanation offered by the authors is that these metals may have parallel mechanisms of action that work competitively [80]. Co-exposure to $\mathrm{Pb}$ and $\mathrm{As}$ in soil at maternal residences during pregnancy was estimated using geospatial statistical models then regressed in relation to intellectual disability (ID) in $\sim 8$ to 12 year olds [77]. Joint exposure to high $\mathrm{Pb}$ and $\mathrm{As}$ in the prenatal period was associated with a higher probability of ID among children with normal birth weight for gestational age compared to low joint exposure or high exposure to one metal at a time.

Two-way interactions between $\mathrm{Mn}$ and either selenium or As have been explored in at least three studies [75, 78, 79]. Selenium, an essential nutrient like $\mathrm{Mn}$, is an antioxidant and was found to be protective against adverse effects of excess prenatal Mn exposure, measured in cord serum, on scores of the NBNA [79]. Arsenic, which may be neurotoxic on its own, did not interact with $\mathrm{Mn}$ on intellectual function or academic achievement in two cross-sectional studies in Bangladesh, where 8- to 11-year-old children were exposed to elevated levels of naturally occurring $\mathrm{As}$ and $\mathrm{Mn}$ in drinking water $[75,78]$. If true, the lack of a Mn-As interaction may indicate that these metals have distinct biologic mechanisms of toxicity. It is also possible that the main effects of these metals at higher exposure levels, such as those observed in these Bangladeshi cohorts, overwhelm any interactive effect. Further, at lower exposure levels, a Mn-As interaction has been previously reported on IQ, verbal learning, and memory among 11- to 13-year-old US children [84].

The two studies that estimated neurodevelopmental effects of more than two metals at a time $\left[16 \bullet, 76^{\bullet}\right]$ were heterogeneous in their populations, study design, biomarkers used to estimate exposure, neurodevelopmental outcomes examined, and statistical methods applied to evaluate combined effects. With prospective data from an ongoing study of Bangladeshi children, Bayesian kernel machine regression was used to flexibly model the exposure-response function of an arseniclead-manganese mixture [16 ${ }^{\circ}$. The authors reported an inverted U-shaped association between cord blood $\mathrm{Mn}$ and the 2-year motor composite score from the BSID-III at the median cord blood $\mathrm{Pb}$ level, though this effect was only observed in the middle levels of As exposure. No biological explanation was provided for this finding (as this was a biostatistic method paper), but an inverted $U$ relationship that reflects Mn essentiality at low levels and toxicity at high levels could be disrupted by higher levels of a co-occurring toxicant such as As. Cross-sectional associations of $\mathrm{As}, \mathrm{Pb}, \mathrm{Mn}$, and $\mathrm{Cd}$ with scores on the BSID-III were examined among preschool children in Uruguay [76 ${ }^{\bullet}$. Children were assigned to four latent clusters (low metal exposure, low-to-moderate metal exposure, high lead and cadmium, and high metal exposure) according to their metal exposure characteristics, and these clusters were modeled in relation to cognitive and language scores. No associations were observed, although the sample size was small ( $n=92$ with imputed data) and the variability around estimates was large.

\section{Behavioral Effects}

We identified five articles that reported metal mixture effects on behavioral outcomes $\left[42,62,71,85,866^{\bullet}\right.$. One study used a prospective design to evaluate prenatal and childhood metal mixture exposures in relation to impulsivity [85]; three studies used a cross-sectional design to investigate ADHD, ADHDlike behaviors, and other externalizing and internalizing behaviors [42, 62, 75]; and one case-control study examined ASD [86॰]. Metal exposure was estimated with biomarkers 
(blood, hair, and urine) as well as environmental samples (drinking water, air, soil).

Four studies evaluated binary combinations of metals [42, $62,71,85]$. Again, $\mathrm{Pb}$ was the most commonly examined metal with respect to interactions with one other metal $(\mathrm{Hg}$, $\mathrm{Cd}$, and $\mathrm{Mn}$ ). Prenatal $\mathrm{Pb}$ exposure, measured in umbilical cord blood, was associated with increased impulsivity among 9 to 13 year olds that was enhanced in the presence of higher $\mathrm{Hg}$ exposure [85]. This synergistic $\mathrm{Pb}-\mathrm{Hg}$ interaction on response inhibition is in contrast to the antagonistic $\mathrm{Pb}-\mathrm{Hg}$ interaction observed for cognition among children 7 and 14 years of age from the Faroe Islands [80]. No interactions between $\mathrm{Pb}$ and $\mathrm{Mn}$ were observed on self-reported behavior on the Conners-Wells' Scale among Italian adolescents [62], and interactions between $\mathrm{Pb}$ and $\mathrm{Cd}$ on ADHD among 6 to 15 year olds were not statistically significant [42]. In a crosssectional study of 8-to 11 year olds from Bangladesh, no statistical interactions were observed between $\mathrm{Mn}$ and As exposure on behavior [71].

Only one study estimated joint effects of more than two metals on behavior [86 ${ }^{\bullet}$. Roberts et al. assessed perinatal exposure to ambient concentrations of antimony, $\mathrm{As}, \mathrm{Cd}$, chromium, $\mathrm{Pb}, \mathrm{Mn}, \mathrm{Hg}$, and nickel, as well as other nonmetal pollutants, based on US EPA dispersion models [86 ${ }^{\circ}$. Associations between ASD and overall metal exposure were estimated in two ways: An overall measure of metal concentrations was derived by summing the quintile category score for each metal, and an overall estimate of association was derived by pooling odds ratios (ORs) from individual metals. The overall metal score and the pooled OR for metals were both positively associated with ASD, suggesting that perinatal exposure to multiple metals may increase the risk of ASD.

\section{Recommendations for Future Directions}

There is a growing body of literature supporting the neurodevelopmental toxicity of $\mathrm{Mn}$ exposure. For $\mathrm{Cd}$, the literature is sparser, but there is some evidence that $\mathrm{Cd}$ may be neurotoxic to children and more research is needed. The metal mixture literature is beginning to identify interactive and joint effects on cognition; however, there is a general lack of studies on behavioral effects. There remain many research gaps, including selecting proper exposure biomarkers, addressing the importance of exposure timing and the role of life stage on neurobehavioral testing, collecting prospective data to evaluate persistence of possible effects, and implementing novel statistical modeling to disentangle mixture effects. It may be unrealistic for the research community to systematically address all of these issues, but the role of each should be taken into consideration when assessing new studies on these metals.

\section{Selection of Biomarkers}

No single biomarker clearly stands out when studying $\mathrm{Cd}$ or $\mathrm{Mn}$ toxicity. In the studies reviewed, Cd was measured most often in blood or urine and less frequently in hair or placenta. Once steady state is reached, $\mathrm{Cd}$ in blood has a half-life of over 10 years [18] and represents recent as well as past exposure. Urine $\mathrm{Cd}$ reflects cumulative exposure as well as the kidney-specific burden [87]. In the Mn studies reviewed, Mn was measured in blood, hair, drinking water, and tooth enamel. Blood Mn generally reflects recent and acute exposure, whereas hair reflects past exposure and integrates exposure over several months. Because $\mathrm{Mn}$ is an essential nutrient, blood $\mathrm{Mn}$ is under tight regulatory control. Blood Mn may then only reflect body burden in relatively extreme cases of overload or deficiency. Evidence from occupational studies shows that high workplace Mn exposure correlates with blood Mn among subjects [88], although this has not been consistent and some data suggest that the utility of blood $\mathrm{Mn}$ as an exposure biomarker may depend on the exposure parameters [89]. Nevertheless, many studies have found relationships between blood $\mathrm{Mn}$ and neurological outcomes. Although not represented in the literature herein, toenail $\mathrm{Mn}$ also represents a biomarker of cumulative exposure that integrates exposure from 7 months to 1 year in the past [90]. Mn is also found in teeth, which possess daily growth rings analogous to those found in trees and allow for more precise determination of exposure timing. In contrast to tooth enamel, dentine Mn has recently been validated, reflects a child's longitudinal metal exposure prenatally through 6-12 years of life [91], and correlates with environmental measures [92] and with cord blood [73]. Therefore, dentine (tooth) Mn holds promise as a sensitive and precise biomarker of exposure in early life [91].

\section{Timing of Exposure and Sensitive Developmental Windows}

Neurodevelopmental processes such as neurogenesis, anatomic organization of neurons/synapses, synaptogenesis, and synaptic pruning are at their peak during fetal life and childhood relative to adult life [93]. Most of these processes also occur through adolescence as well. Central nervous system development underlies the sensitivity of children to toxic chemicals [94]. It is therefore critical to consider timing of environmental exposures because certain developmental periods may be more sensitive to the effects of environmental exposures than others. For example, animal studies have shown that prenatal Mn exposure had greater effects on markers of inflammatory neurotoxicity (reduced glutathione and metallothionein gene expression) among neonatal rats compared to older rats [95, 96].

These issues are further complicated if one considers mixture effects, as mixed effects are just as likely as main effects 
to be dependent on exposure timing and each chemical may act differentially depending on the age of exposure. In this regard, laser ablation-inductively coupled plasma-mass spectrometry analysis of deciduous teeth holds particular promise [97]. New methods that can objectively reconstruct past exposure to multiple metals and their mixtures will allow researchers to overcome many of the research gaps noted above. More research is needed with prospective exposure data that can assess the role of windows of susceptibility, especially for behavioral outcomes. Specific knowledge gaps in the mixture literature include a lack of data among young (1-3 years old) children and adolescents ( $\sim 15-19$ year olds).

Development not only induces sensitivity to chemicals but also plays a role in interpreting exposure biomarkers. For example, blood $\mathrm{Mn}$ is highest in infancy and remains higher than the average adult level until approximately 12 years of age and then gradually declines through adulthood [98]. These agespecific shifts in blood Mn levels are significantly more pronounced among females than males [98]. Future research estimating body burden of Mn should consider these age- and gender-specific differences in exposure biomarkers and the role of $\mathrm{Mn}$ as an essential nutrient during development.

\section{The Role of Life Stage on Neurobehavioral Testing}

Unlike adults, nearly all behavioral assessment tools in children are age specific. This is because not all functions are present at birth but are expressed at different life stages and deficits from early life exposure may not be measurable for years. IQ cannot be assessed in a toddler, for example. Neonatal phenotypes have a large amount of measurement error, simply because neonates have limited variability in their behaviors. Associations with toxic exposures among neonates are generally less stable over time than relationships seen at older ages. This property, while less pronounced, holds when assessing cognition and behavior in children and adolescents. Moreover, many traits are not expressed until a specific life stage. For example, internalizing behaviors are more easily measured in a 9 year old than in a toddler but are most prevalent in adolescence (i.e., traits themselves are life stage specific). If we are to understand the role of exposure timing, future work should develop better neurobehavioral assessment tools at younger ages to allow for repeated measurements of functional domains at multiple life stages.

\section{Complex Statistical Modeling}

Although an increasing number of epidemiologic studies are considering metal co-exposure as a potential confounder, fewer studies have evaluated joint, interactive, or modifying effects of higher order co-exposure (i.e., three or more chemicals). This may be due, at least in part, to challenges related to choosing a biomarker or measure of exposure that is appropriate for all metals of interest, difficulties related to collecting multiple biomarkers, the cost of analyzing multiple metals and/or multiple exposure measures, and statistical challenges such as limited power and analysis of highly correlated data [14]. In the present mixture literature, there is little consistency in terms of metals evaluated, measures of exposure used for each metal, and statistical approach employed for analyzing joint or interactive effects. Methodologies to address higher order mixtures such as weighted quantile sum regression [15] and Bayesian kernel machine regression [16•] will help to address some knowledge gaps.

\section{Conclusion}

This review of the current literature suggests that early life $\mathrm{Cd}$ and $\mathrm{Mn}$ exposure leads to reductions in childhood cognitive ability. There does not appear to be evidence of a relationship between $\mathrm{Cd}$ and ADHD, but a small number of studies provide suggestive evidence of a relationship with other negative behavioral outcomes. There is suggestive evidence of a link between Mn with ADHD, autism, and other behavioral outcomes. Limited epidemiologic evidence suggests that joint exposure to multiple metals may have different, and in many cases worse, effects on neurodevelopment and cognition than effects of individual metals, in particular when the mixture includes $\mathrm{Pb}$.

Acknowledgments This work was supported, in part, by funding from the NIH/NIEHS: P42ES016454, P30ES23515, R01ES013744, R01ES020268, R01ES021357, and R00ES022986.

\section{Compliance with Ethics Guidelines}

Conflict of Interest Alison P. Sanders, Birgit Claus Henn, and Robert $\mathrm{O}$. Wright declare that they have no conflict of interest.

Human and Animal Rights and Informed Consent This article does not contain any studies with human or animal subjects performed by any of the authors.

\section{References}

Papers of particular interest, published recently, have been highlighted as:

- Of importance

•• Of major importance

1. Laborde A, et al. Children's health in Latin America: the influence of environmental exposures. Environ Health Perspect. 2014. doi: 10.1289/ehp. 1408292

2. CDC. Fourth national report on human exposure to environmental chemicals, updated tables, D.o.H.a.H. Services, editor. 2013. Atlanta: US Department of Health and Human Services. 
3. Lanphear BP, Roghmann KJ. Pathways of lead exposure in urban children. Environ Res. 1997;74(1):67-73.

4. Ziegler EE et al. Absorption and retention of lead by infants. Pediatr Res. 1978;12(1):29-34.

5. Malcoe LH et al. Lead sources, behaviors, and socioeconomic factors in relation to blood lead of Native American and white children: a community-based assessment of a former mining area. Environ Health Perspect. 2002;110 Suppl 2:221-31.

6. Lidsky TI, Schneider JS. Lead neurotoxicity in children: basic mechanisms and clinical correlates. Brain. 2003;126(Pt 1):5-19.

7. Grosse $\mathrm{SD}$ et al. Economic gains resulting from the reduction in children's exposure to lead in the United States. Environ Health Perspect. 2002;110(6):563-9.

8. Trasande L, Liu Y. Reducing the staggering costs of environmental disease in children, estimated at $\$ 76.6$ billion in 2008. Health Aff (Millwood). 2011;30(5):863-70.

9. Landrigan PJ et al. Environmental pollutants and disease in American children: estimates of morbidity, mortality, and costs for lead poisoning, asthma, cancer, and developmental disabilities. Environ Health Perspect. 2002;110(7):721-8.

10. Tyler CR, Allan AM. The effects of arsenic exposure on neurological and cognitive dysfunction in human and rodent studies: a review. Curr Environ Health Rep. 2014;1:132-47.

11. Tolins M, Ruchirawat M, Landrigan P. The developmental neurotoxicity of arsenic: cognitive and behavioral consequences of early life exposure. Ann Glob Health. 2014;80(4):303-14.

12. $\mathrm{Hu} \mathrm{H}$, Shine J, Wright RO. The challenge posed to children's health by mixtures of toxic waste: the Tar Creek superfund site as a casestudy. Pediatr Clin North Am. 2007;54(1):155-75.

13. Sexton $\mathrm{K}$ et al. Using biologic markers in blood to assess exposure to multiple environmental chemicals for inner-city children 3-6 years of age. Environ Health Perspect. 2006;114(3):453-9.

14. Claus Henn B, Coull BA, Wright RO. Chemical mixtures and children's health. Curr Opin Pediatr. 2014;26(2):223-9.

15. Gennings $\mathrm{C}$ et al. A cohort study evaluation of maternal PCB exposure related to time to pregnancy in daughters. Environ Health. 2013;12(1):66.

16. Bobb JF, et al. Bayesian kernel machine regression for estimating the health effects of multi-pollutant mixtures. Biostatistics. 2014. doi: 10.1093/biostatistics/kxu058. This study introduces an approach to flexibly model exposure-response functions of chemical mixtures. It demonstrates the use of this method to evaluate associations of a mixture of three metals with motor development. It is one of few studies published to date that have evaluated joint effects of more than two metals at a time on neurodevelopment.

17. Carlin DJ et al. Unraveling the health effects of environmental mixtures: an NIEHS priority. Environ Health Perspect. 2013;121(1):A6-8.

18. Jarup L, Akesson A. Current status of cadmium as an environmental health problem. Toxicol Appl Pharmacol. 2009;238(3):201-8.

19. ATSDR. Toxicological profile for cadmium. 2008 [cited 2011 July 1]. Available from http://www.atsdr.cdc.gov/toxprofiles/tp5. pdf

20. Rudge CV et al. The placenta as a barrier for toxic and essential elements in paired maternal and cord blood samples of South African delivering women. J Environ Monit. 2009;11(7):1322-30.

21. Salpietro CD et al. Cadmium concentration in maternal and cord blood and infant birth weight: a study on healthy non-smoking women. J Perinat Med. 2002;30(5):395-9.

22. Taylor CM, Golding J, Emond AM. Lead, cadmium and mercury levels in pregnancy: the need for international consensus on levels of concern. J Dev Origins Health Dis. 2014;5(1):16-30.

23. Eklund G, Oskarsson A. Exposure of cadmium from infant formulas and weaning foods. Food Addit Contam. 1999;16(12):509-19.
24. Dabeka $\mathrm{R}$ et al. Lead, cadmium and aluminum in Canadian infant formulae, oral electrolytes and glucose solutions. Food Addit Contam Part A Chem Anal Control Expo Risk Assess. 2011;28(6):744-53.

25. Winiarska-Mieczan A. Cadmium, lead, copper and zinc in breast milk in Poland. Biol Trace Elem Res. 2014;157(1):36-44.

26. Dobson AW, Erikson KM, Aschner M. Manganese neurotoxicity. Ann N Y Acad Sci. 2004;1012:115-28.

27. Erikson KM et al. Manganese neurotoxicity: a focus on the neonate. Pharmacol Ther. 2007;113(2):369-77.

28. ATSDR. Toxicological profile for manganese. 2008 [cited 2011 July 1].

29. Davidsson L et al. Manganese retention in man: a method for estimating manganese absorption in man. Am J Clin Nutr. 1989;49(1): 170-9.

30. Davidsson $\mathrm{L}$ et al. Intrinsic and extrinsic labeling for studies of manganese absorption in humans. J Nutr. 1988;118(12):1517-21.

31. Ramirez F. Pesticides imports in Costa Rica 2007-2009: report for the REPCar Project. In: Central American Institute for Studies on Toxic Substances, 2010. Costa Rica: Universidad Nacional de Costa Rica.

32. U.S. Environmental Protection Agency. Pesticides industry sales and usage: 2006 and 2007 market estimates. Washington: U.S. Environmental Protection Agency; 2011.

33. Krachler M, Rossipal E, Micetic-Turk D. Trace element transfer from the mother to the newborn-investigations on triplets of colostrum, maternal and umbilical cord sera. Eur J Clin Nutr. 1999;53(6): 486-94.

34. Guan $\mathrm{H}$ et al. Manganese concentrations in maternal and umbilical cord blood: related to birth size and environmental factors. Eur J Public Health. 2014;24(1):150-7.

35. Kopp RS et al. Partition of metals in the maternal/fetal unit and lead-associated decreases of fetal iron and manganese: an observational biomonitoring approach. Arch Toxicol. 2012;86(10):157181.

36. Krachler $\mathrm{M}$ et al. Concentrations of selected trace elements in human milk and in infant formulas determined by magnetic sector field inductively coupled plasma-mass spectrometry. Biol Trace Elem Res. 2000;76(2):97-112.

37. Cockell KA, Bonacci G, Belonje B. Manganese content of soy or rice beverages is high in comparison to infant formulas. J Am Coll Nutr. 2004;23(2):124-30.

38. Bouchard MF. Manganese in drinking water: Bouchard responds. Environ Health Perspect. 2011;119(6):A241.

39. Elsner RJ, Spangler JG. Neurotoxicity of inhaled manganese: public health danger in the shower? Med Hypotheses. 2005;65(3):60716.

40. Al-Saleh I et al. Birth outcome measures and maternal exposure to heavy metals (lead, cadmium and mercury) in Saudi Arabian population. Int J Hyg Environ Health. 2014;217(2-3):205-18.

41. Cao Y et al. Postnatal cadmium exposure, neurodevelopment, and blood pressure in children at 2, 5, and 7 years of age. Environ Health Perspect. 2009;117(10):1580-6.

42. Ciesielski $\mathrm{T}$ et al. Cadmium exposure and neurodevelopmental outcomes in U.S. children. Environ Health Perspect. 2012;120(5):758 63.

43. Forns $\mathrm{J}$ et al. Exposure to metals during pregnancy and neuropsychological development at the age of 4 years. Neurotoxicology. 2014;40:16-22.

44. Garcia-Esquinas E et al. Lead, mercury and cadmium in umbilical cord blood and its association with parental epidemiological variables and birth factors. BMC Public Health. 2013;13:841.

45. Jeong $\mathrm{KS}$ et al. Performance IQ in children is associated with blood cadmium concentration in early pregnancy. J Trace Elem Med Biol. 2015;30:107-11. 
46. Kim Y et al. Prenatal lead and cadmium co-exposure and infant neurodevelopment at 6 months of age: the Mothers and Children's Environmental Health (MOCEH) study. Neurotoxicology. 2013;35:15-22.

47. Kippler M et al. Early-life cadmium exposure and child development in 5-year-old girls and boys: a cohort study in rural Bangladesh. Environ Health Perspect. 2012;120(10):1462-8.

48. Rodriguez-Barranco $\mathrm{M}$ et al. Cadmium exposure and neuropsychological development in school children in southwestern Spain. Environ Res. 2014;134:66-73.

49. Tian LL et al. Effects of gestational cadmium exposure on pregnancy outcome and development in the offspring at age 4.5 years. Biol Trace Elem Res. 2009;132(1-3):51-9.

50. Bao QS et al. Behavioural development of school-aged children who live around a multi-metal sulphide mine in Guangdong province, China: a cross-sectional study. BMC Public Health. 2009;9: 217.

51. Kim S et al. Lead, mercury, and cadmium exposure and attention deficit hyperactivity disorder in children. Environ Res. 2013;126: $105-10$.

52. Sioen I et al. Prenatal exposure to environmental contaminants and behavioural problems at age 7-8 years. Environ Int. 2013;59:22531.

53. Szkup-Jablonska M et al. Effects of blood lead and cadmium levels on the functioning of children with behaviour disorders in the family environment. Ann Agric Environ Med. 2012;19(2):241-6.

54. Yousef $\mathrm{S}$ et al. Attention deficit hyperactivity disorder and environmental toxic metal exposure in the United Arab Emirates. J Trop Pediatr. 2011;57(6):457-60

55. Bhang SY et al. Relationship between blood manganese levels and children's attention, cognition, behavior, and academic performance-a nationwide cross-sectional study. Environ Res. 2013;126:9-16.

56. Bouchard MF et al. Intellectual impairment in school-age children exposed to manganese from drinking water. Environ Health Perspect. 2011;119(1):138-43.

57. Carvalho $\mathrm{CF}$ et al. Elevated airborne manganese and low executive function in school-aged children in Brazil. Neurotoxicology. 2014;45:301-8.

58. Claus Henn B et al. Early postnatal blood manganese levels and children's neurodevelopment. Epidemiology. 2010;21(4):433-9.

59. do Nascimento SN et al. Cognitive deficits and ALA-D-inhibition in children exposed to multiple metals. Environ Res. 2015;136: 387-95.

60. Kim Y et al. Co-exposure to environmental lead and manganese affects the intelligence of school-aged children. Neurotoxicology. 2009;30(4):564-71.

61. Lin CC et al. In utero exposure to environmental lead and manganese and neurodevelopment at 2 years of age. Environ Res. 2013;123:52-7.

62. Lucchini RG et al. Inverse association of intellectual function with very low blood lead but not with manganese exposure in Italian adolescents. Environ Res. 2012;118:65-71.

63. Menezes-Filho JA et al. Elevated manganese and cognitive performance in school-aged children and their mothers. Environ Res. 2011;111(1):156-63.

64. Rink SM et al. Associations between hair manganese levels and cognitive, language, and motor development in preschool children from Montevideo, Uruguay. Arch Environ Occup Health. 2014;69(1):46-54.

65. Riojas-Rodriguez $\mathrm{H}$ et al. Intellectual function in Mexican children living in a mining area and environmentally exposed to manganese. Environ Health Perspect. 2010;118(10):1465-70.

66. Torres-Agustin R et al. Effect of environmental manganese exposure on verbal learning and memory in Mexican children. Environ Res. 2013;121:39-44.
67. Yu XD et al. Prenatal exposure to manganese at environment relevant level and neonatal neurobehavioral development. Environ Res. 2014;133:232-8.

$68 . \cdots$ Chung SE, et al. Maternal blood manganese and early neurodevelopment: the Mothers and Children's Environmental Health (MOCEH) study. Environ Health Perspect, 2015. [Epub ahead of print.] This study used penalized spline models to demonstrate an inverted $U$-shaped association between prenatal Mn exposure and mental development at 6 months of age. The authors identified a threshold of $24-28 \mu \mathrm{g} / \mathrm{L}$ maternal blood $\mathrm{Mn}$, above which deficits in the mental development index were observed.

69. Abdullah MM et al. Heavy metal in children's tooth enamel: related to autism and disruptive behaviors? J Autism Dev Disord. 2012;42(6):929-36.

70. Farias AC et al. Manganese in children with attention-deficit/hyperactivity disorder: relationship with methylphenidate exposure. J Child Adolesc Psychopharmacol. 2010;20(2):113-8.

71. Khan $\mathrm{K}$ et al. Manganese exposure from drinking water and children's classroom behavior in Bangladesh. Environ Health Perspect. 2011;119(10):1501-6.

72. Beaudin SA et al. Oral methylphenidate alleviates the fine motor dysfunction caused by chronic postnatal manganese exposure in adult rats. Toxicol Sci. 2015;144(2):318-27.

73. Arora $\mathrm{M}$ et al. Determining fetal manganese exposure from mantle dentine of deciduous teeth. Environ Sci Technol. 2012;46(9):511825.

74. Claus Henn B et al. Associations of early childhood manganese and lead coexposure with neurodevelopment. Environ Health Perspect. 2012;120(1):126-31.

75. Khan $\mathrm{K}$ et al. Manganese exposure from drinking water and children's academic achievement. Neurotoxicology. 2012;33(1):91-7.

76. Kordas K et al. Patterns of exposure to multiple metals and associations with neurodevelopment of preschool children from Montevideo, Uruguay. J Environ Public Health. 2015;2015: 493471. This study assesses joint effects of four metals on cognitive and language scores using latent class analysis. It is one of few studies published to date that have evaluated joint effects of more than two metals at a time on neurodevelopment.

77. McDermott $\mathrm{S}$ et al. Probability of intellectual disability is associated with soil concentrations of arsenic and lead. Chemosphere. 2011;84(1):31-8.

78. Wasserman GA et al. Arsenic and manganese exposure and children's intellectual function. Neurotoxicology. 2011;32(4):450-7.

79. Yang $X$ et al. Selenium protects neonates against neurotoxicity from prenatal exposure to manganese. PLoS One. 2014;9(1), e86611.

80. Yorifuji $\mathrm{T}$ et al. Prenatal exposure to lead and cognitive deficit in 7and 14-year-old children in the presence of concomitant exposure to similar molar concentration of methylmercury. Neurotoxicol Teratol. 2011;33(2):205-11.

81. Kalia K, Chandra SV, Viswanathan PN. Effect of 54Mn and lead interaction on their binding with tissue proteins: in vitro studies. Ind Health. 1984;22(3):207-18.

82. Devoto $\mathrm{P}$ et al. Lead intoxication during intrauterine life and lactation but not during adulthood reduces nucleus accumbens dopamine release as studied by brain microdialysis. Toxicol Lett. 2001;121(3):199-206.

83. Eriksson $\mathrm{H}$ et al. Receptor alterations in manganese intoxicated monkeys. Arch Toxicol. 1992;66(5):359-64.

84. Wright RO et al. Neuropsychological correlates of hair arsenic, manganese, and cadmium levels in school-age children residing near a hazardous waste site. Neurotoxicology. 2006;27(2):210-6.

85. Boucher $\mathrm{O}$ et al. Response inhibition and error monitoring during a visual go/no-go task in Inuit children exposed to lead, polychlorinated biphenyls, and methylmercury. Environ Health Perspect. 2012;120(4):608-15. 
86. Roberts AL et al. Perinatal air pollutant exposures and autism spectrum disorder in the children of Nurses' Health Study II participants. Environ Health Perspect. 2013;121(8):978-84. This is the only study published to date of which we are aware that has evaluated joint effects of more than two metals with a behavioral outcome (autism spectrum disorder).

87. Nordberg GF et al. Biological monitoring of cadmium exposure and renal effects in a population group residing in a polluted area in China. Sci Total Environ. 1997;199(1-2): $111-4$.

88. Ellingsen DG et al. Air exposure assessment and biological monitoring of manganese and other major welding fume components in welders. J Environ Monit. 2006;8(10):1078-86.

89. Smith $\mathrm{D}$ et al. Biomarkers of Mn exposure in humans. Am J Ind Med. 2007;50(11):801-11.

90. Laohaudomchok W et al. Toenail, blood, and urine as biomarkers of manganese exposure. J Occup Environ Med. 2011;53(5):506-10.

91. Arora $\mathrm{M}$ et al. Spatial distribution of manganese in enamel and coronal dentine of human primary teeth. Sci Total Environ. 2011;409(7):1315-9.
92. Gunier RB et al. Biomarkers of manganese exposure in pregnant women and children living in an agricultural community in California. Environ Sci Technol. 2014;48(24):14695-702.

93. Tau GZ, Peterson BS. Normal development of brain circuits. Neuropsychopharmacology. 2010;35(1):147-68.

94. Rice D, Barone Jr S. Critical periods of vulnerability for the developing nervous system: evidence from humans and animal models. Environ Health Perspect. 2000;108 Suppl 3:511-33.

95. Erikson $\mathrm{KM}$ et al. Airborne manganese exposure differentially affects end points of oxidative stress in an age- and sex-dependent manner. Biol Trace Elem Res. 2004;100(1):49-62.

96. Erikson $\mathrm{KM}$ et al. Alterations of oxidative stress biomarkers due to in utero and neonatal exposures of airborne manganese. Biol Trace Elem Res. 2006;111(1-3):199-215.

97. Hare $\mathrm{D}$ et al. Elemental bio-imaging of trace elements in teeth using laser ablation-inductively coupled plasma-mass spectrometry. J Dent. 2011;39(5):397-403.

98. Oulhote Y, Mergler D, Bouchard MF. Sex- and age-differences in blood manganese levels in the U.S. general population: National Health and Nutrition Examination Survey 2011-2012. Environ Health. 2014;13:87. 